\title{
Comparaison, dans des conditions courantes, de l'efficacité de quatre méthodes de nettoyage des machines à traire
}

\author{
par \\ Y. M. CHATELIN*; J. RICHARD** \\ avec la collaboration de : \\ Marie-José ESCUDE*, Christine BRAQUEHAYE**, \\ J. P. CHALARD ${ }^{* * *}$ et J. P. LENEVANEN****
}

\section{R és u m é}

On a comparé, pendant plus d'un an dans 23 exploitations ordinaires, les quatre méthodes de nettoyage suivantes : a) la méthode utilisant, deux fois par jour, de l'eau chaude acidifiée à 95-98 $\mathrm{C}$ (E.C.A.) ; b) une variante de cette dernière consistant en un simple rinçage à l'eau froide après la traite du soir (E.C.A. simplifiée) ; c) la méthode classique, consistant en un nettoyage en circuit fermé, deux fois par jour, avec une solution chaude $\left(50-60^{\circ} \mathrm{C}\right)$ d'un détergent alcalin; d) une variante de cette dernière dans laquelle, on alterne quotidiennement une solution alcaline et une solution acide (alternance).

* I.T.E.B. : 149, rue de Bercy - 75595 Paris cedex 12.

$*$ I.N.R.A. : Laboratoire de Microbiologie Laitière et de Génie Alimentaire 78350 Jouy-en-Josas.

*** Contrôle Laitier et Services aux Eleveurs de la Sarthe, "La Futaie » Rouillon - 72700 Allonnes.

**** Union Laitière de la Meuse, 23 bis, rue des Frères-Boulhaut, B.P. 149 - 55104 Verdun. 
Le rinçage des installations de traite avec de l'eau désinfectée par le chlore a été employé pour déterminer le « pouvoir contaminant " de celles-ci. Les échantillons prélevés ont été soumis au dénombrement des flores totale, thermorésistante, psychrotrophe, psychrotrophe résistante à la pénicilline (composée essentiellement de Pseudomonas), et des bactéries coliformes.

En moyenne, la méthode E.C.A. donnait régulièrement les résultats les meilleurs, mais toutefois moins bons que dans des fermes expérimentales ou soumises à un contrôle périodique. L'alternance conduisait aux résultats les moins satisfaisants et les plus variables, ceux obtenus avec la méthode classique et la méthode E.C.A. simplifiée, se situant en position moyenne. Cependant, du fait de la variabilité des résultats entre fermes recourant à la même méthode de nettoyage et à l'intérieur de certaines exploitations, ces différences n'étaient généralement pas statistiquement significatives. Les méhodes basées sur l'utilisation de l'eau très chaude (E.C.A. ou E.C.A. simplifiée) favorisaient l'implantation de micro-organismes thermorésistants dans le matériel alors que les autres méthodes permettaient plutôt la prolifération de la flore à Gram négatif (bactéries coliformes et flores psychrotrophes).

Cette étude souligne la nécessité d'évaluer l'efficacité des méthodes de nettoyage sur une période suffisamment longue et dans des conditions réellement pratiques.

Mots clés :

Machines à traire - Nettoyage - Efficacité - Evaluation.

Titre abrégé :

Efficacité du nettoyage des machines à traire.

\section{S u m m a r y}

\section{COMPARISON OF THE EFFICACY}

OF FOUR MILKING MACHINE CLEANING METHODS

UNDER UNSUPERVISED FIELD CONDITIONS

The four following cleaning methods (i) acidified boiling water (A.B.W.) twice a day, (ii) A.B.W. once a day with a cold water rinsing after the evening milking, (iii) hot alcaline circulation (H.A.C.) after each milking and (iv) alternatively H.A.C. and hot acid circulation were compared in a one year survey in 23 unsupervised farms. The rinsing method (pulsation rinse of the entire system with 
disinfected water) was used as bacteriological assessement of the maximum possible level of microbial contamination of the milk by the milking machines.

The samples were submitted to the following enumeration: total, thermoduric, psychrotrophic, penicillin-resistant psychrotrophic and coliform counts.

On the average, the A.B.W. method gave the best results but these were not as good as those obtained in experimental or supervised farms; (iv) gave the less satisfactory and reproducible results. (ii) and (iii) gave intermediate ones. However, due to variations between farms using the same method, and sometime within farms, these differences generally were not statistically significant.

In general, the A.B.W. (basal or simplified) methods tended to cause a build-up of the heat resistant flora and the hot methods favoured the multiplication of Gram negative bacteria (coliforms and psychrotrophic counts) in the milking machines.

This study emphasizes the need for an assessement of the long term efficacy of the cleaning methods in truly practical conditions. Recommendations are made for such a study.

Key words:

Milking machines - Cleaning - Efficacy - Evaluation.

\section{INTRODUCTION}

La plupart des méthodes de nettoyage des machines à traire actuellement recommandées ont été mises au point dans des étables expérimentales. Leur efficacité, en pratique, a été généralement vérifiée au cours d'essais plus ou moins longs conduits dans plusieurs fermes habituellement soumises à un contrôle régulier (Clough et al., 1965 ; Palmer et al., 1970 ; Richard, 1974 ; Sinclair et Parry, 1978 ; Richard, 1981 a).

L'efficacité à long terme de ces méthodes, dans des exploitations ne bénéficiant pas d'une surveillance particulière, a rarement été déterminée ou l'a été uniquement par le biais de l'évaluation de la qualité bactériologique du lait produit dans ces exploitations. Or, nous avons montré que l'installation de traite n'est pas toujours la source principale de contamination microbienne du lait (Chatelin 
et Richard, 1981; Piton et Richard, 1982. D'autre part, il est utile de connaître non seulement l'importance de la flore totale provenant du matériel de traite, mais aussi l'apport de celui-ci en microorganismes d'intérêt technologique : flore psychrotrophe ou mieux psychrotrophe résistante à la pénicilline (Richard, 1981 b), flore thermorésistante et bactéries coliformes.

En outre, soit pour des raisons économiques, soit qu'on juge a priori, ces variantes plus efficaces, des variantes aux méthodes de nettoyage recommandées tendent à s'implanter dans les fermes, le plus souvent sans que leurs performances aient été établies de façon précise.

Ces diverses raisons nous ont amenés à procéder, selon une méthodologie appropriée, à une évaluation, dans les conditions pratiques, de l'efficacité des méthodes de nettoyage les plus utilisées actuellement en France. Cette méthodologie est basée sur la détermination directe du « pouvoir contaminant maximum » des installations de traite (Richard, 1981 c), une analyse bactériologique aussi complète que possible des échantillons prélevés et l'étude de la variabilité des résultats obtenus.

\section{MATERIEL ET METHODES}

\section{Choix des exploitations}

De mars 1978 à juin 1979, 23 exploitations (8 dans le département de la Meuse, 15 dans celui de la Sarthe) ont été visitées à 3 ou 4 reprises (voire 5 pour certaines d'entre elles).

Ces exploitations disposaient toutes d'une salle de traite avec lactoduc : elles ont été retenues parce que les méthodes de nettoyage que nous désirions étudier paraissaient y être appliquées correctement et régulièrement et qu'en outre, les installations de traite concernées ne présentaient pas de défauts visibles de conception ou de montage. Les plus modestes d'entre elles (et les plus nombreuses dans notre échantillon) étaient de type "épi » 2 fois 3 stalles simple équipement, les plus importantes, de type " épi » 2 fois 5 stalles double équipement.

\section{Méthodes de nettoyage}

Le nettoyage du matériel de traite était réalisé selon l'une des méthodes suivantes : 
Méthode à l'eau chaude acidifiée (E.C.A.) deux fois par jour suivant la technique décrite par Clough et al (1965) : 6 exploitations.

- Méthode à l'eau chaude acidifiée simplifiée (E.C.A. simplifiée) après la traite du matin; simple rinçage à l'eau froide après celle du soir (ou, dans une ferme, avec une solution à $45^{\circ} \mathrm{C}$ d'un détergent alcalin) : 6 exploitations.

- Méthode classique : après chaque traite, circulation pendant 10 min d'une solution chaude $\left(50\right.$ à $\left.60^{\circ} \mathrm{C}\right)$ d'un détergent alcalin chloré (80 à $100 \mathrm{mg} / \mathrm{l}$ de chlore titrable), l'installation étant, avant et après son nettoyage, rincée à l'eau froide; détartrage périodique au moyen d'un produit acide : 5 exploitations.

- Méthode classique avec alternance de produits (Alternance) : application du nettoyage classique précédemment décrit avec, une traite sur deux, emploi d'une solution chaude $\left(50\right.$ à $\left.60^{\circ} \mathrm{C}\right)$ d'un détergent acide : 6 exploitations.

$\mathrm{Au}$ début de l'étude, nous avons vérifié que les méthodes de nettoyage définies ci-dessus étaient effectivement utilisées et en particulier que dans les exploitations employant de l'eau chaude acidifiée, celle-ci sortait de l'installation de traite pendant au moins 3 min à au moins $75^{\circ} \mathrm{C}$.

\section{Détermination du " pouvoir contaminant maximum " des installations de traite}

Le "pouvoir contaminant maximum " des machines à traire a été déterminé par des rinçages totaux (Richard, 1981 c). Cette technique consiste à faire circuler pendant $5 \mathrm{~min}$, dans l'installation en circuit fermé, les pulsateurs étant en fonctionnement, 20 à $30 \mathrm{l}$ d'eau désinfectée par le chlore. Deux rinçages successifs étaient ainsi réalisés.

\section{Conservation des échantillons}

Les échantillons d'eau de rinçage étaient additionnés de $4 \%$ d'une solution de glycine à $25 \mathrm{~g} / \mathrm{l}$ de façon à éviter une éventuelle destruction des cellules microbiennes (Straka et Stokes 1957); ils étaient ensuite conservés au plus $24 \mathrm{~h}$ à $0^{\circ} \mathrm{C}$.

\section{Numérations microbiennes}

Après avoir traité les échantillons pendant 30 secondes à l'aide d'une turbine tournant à $20000 \mathrm{t} / \mathrm{min}$ (Richard 1980), on a effectué les dénombrements suivants : 
-flore totale sur milieu Plate Count Agar (Difco 479); incubation 3 jours à $30^{\circ} \mathrm{C}$;

à $7^{\circ} \mathrm{C}$;

- flore psychrotrophe sur le même milieu; incubation 10 jours

- flore psychrotrophe résistante à la pénicilline, sur milieu Nutrient Agar (Difco 1) additionné de 100 U.I. de pénicilline par ml ; incubation 10 jours à $7^{\circ} \mathrm{C}$ (Richard, 1981 b) ;

- bactéries coliformes sur milieu Violet Red Bile Agar (Difco 12); incubation 18 à $24 \mathrm{~h}$ à $30^{\circ} \mathrm{C}$;

- flore termorésistante : chauffage pendant 30 min à $63,5^{\circ} \mathrm{C}$ du mélange volume à volume d'eau de rinçage et de lait écrémé stérile (Skim milk Difco à $20 \%$ ), puis dénombrement de la flore thermorésistante sur milieu Plate Count Agar (Difco 479) additionné de $1 \%$ de lait recueilli aseptiquement et pasteurisé; incubation 3 jours à $30^{\circ} \mathrm{C}$.

Tous ces ensemencements, exception faite de ceux destinés au dénombrement des bactéries coliformes, ont été réalisés en surface, avec un appareil Spiral*.

\section{Exploitation des résultats}

Les résultats des dénombrements microbiens, après transformation en logarithmes décimaux, ont fait l'objet d'une analyse discriminante (Lebart et Fénelon, 1973) et d'analyses de la variance de modèles aléatoires déséquilibrés (Snedecor et Cochran, 1967).

\section{RESULTATS ET DISCUSSION}

Le " pouvoir contaminant maximum » des installations de traite a été estimé à partir des résultats des dénombrements microbiens réalisés sur les échantillons prélevés lors des seconds rinçages (Richard, 1981 c).

Le tableau 1 présente, pour chaque méthode de nettoyage, le niveau moyen de chaque groupe microbien, ainsi que les valeurs extrêmes. Les résultats du nettoyage biquotidien à l'eau chaude acidifiée (E.C.A.) se sont révélés nettement moins satisfaisants que ceux obtenus lors d'une étude en étable expérimentale et en exploitations régulièrement suivies (Richard, 1981 a); en revanche, la

* Spiral System Marketing, Interscience, 3, rue de Milan, Paris. 
TABLEAU 1 - TABLE 1

Evaluation du " pouvoir contaminant maximum » des machines à traire (nombre de micro-organismes/ml d'eau au $2^{\mathrm{e}}$ rinçage des installations)

Evaluation of the maximum level of microbial contamination of the milk by the milking machines (bacterial counts $/ \mathrm{ml}$ in the second rinse)

\begin{tabular}{|c|c|c|c|c|c|}
\hline \multirow{2}{*}{$\begin{array}{c}\text { Nombre de } \\
\text { micro-organismes } \\
\text { par ml }\end{array}$} & \multirow{2}{*}{$\begin{array}{l}\text { Paramètres } \\
\text { statistiques }\end{array}$} & \multicolumn{3}{|c|}{ Méthodes de nettoyage } & \multirow{2}{*}{ Alternance } \\
\hline & & E.C.A. & E.C.A. simplifiée & Classique & \\
\hline Flore totale & $\begin{array}{ll}\text { m } & (1) \\
\text { e } & (2)\end{array}$ & $\begin{array}{c}7800 \\
660-71000\end{array}$ & $\begin{array}{c}41000 \\
10-1200000\end{array}$ & $\begin{array}{c}6600 \\
60-300000\end{array}$ & $\begin{array}{c}23000 \\
3000-220000\end{array}$ \\
\hline Flore thermorésistante & $\begin{array}{l}\mathrm{m} \\
\mathrm{e}\end{array}$ & $\begin{array}{c}1100 \\
100-10000\end{array}$ & $\begin{array}{c}6200 \\
100-\quad 150000\end{array}$ & $\begin{array}{ll} & 370 \\
60-\quad 6200\end{array}$ & $\begin{array}{c}1400 \\
100-16000\end{array}$ \\
\hline Flore psychrotrophe & $\begin{array}{l}\mathrm{m} \\
\mathrm{e}\end{array}$ & $\begin{array}{c}700 \\
1-\quad 7400\end{array}$ & $\begin{array}{c}2100 \\
42-\quad 20000\end{array}$ & $\begin{array}{c}1300 \\
46-12000\end{array}$ & $\begin{array}{c}2700 \\
100-14000\end{array}$ \\
\hline $\begin{array}{l}\text { Flore psychrotrophe } \\
\text { résistante à } \\
\text { la pénicilline }\end{array}$ & $\begin{array}{l}\mathrm{m} \\
\mathrm{e}\end{array}$ & $\begin{array}{c}72 \\
5-12000\end{array}$ & $\begin{array}{ll} & 560 \\
13- & 20000\end{array}$ & $\begin{array}{ll} & 400 \\
20- & 17000\end{array}$ & $100-\quad 7900$ \\
\hline $\begin{array}{l}\text { Bactéries } \\
\text { Coliformes }\end{array}$ & $\mathrm{m}$ & $0 .{ }^{5} 250$ & ${ }^{5} 24$ & $\begin{array}{ll} & 17 \\
0- & 1500\end{array}$ & $0--^{53} 560$ \\
\hline
\end{tabular}

(1) Moyenne géométrique de 18 à 23 déterminations (5 à 6 fermes $\times 2$ à 5 visites par ferme).

(2) Valeurs extrêmes observées. 
$\triangle$ Net 1: E.C.A.

$\triangle$ Net 2: E.C.A. simplifiée

Det 3 : CLASSIQUE

- Net 4 : alternance

$\star$ Point moyen du groupe

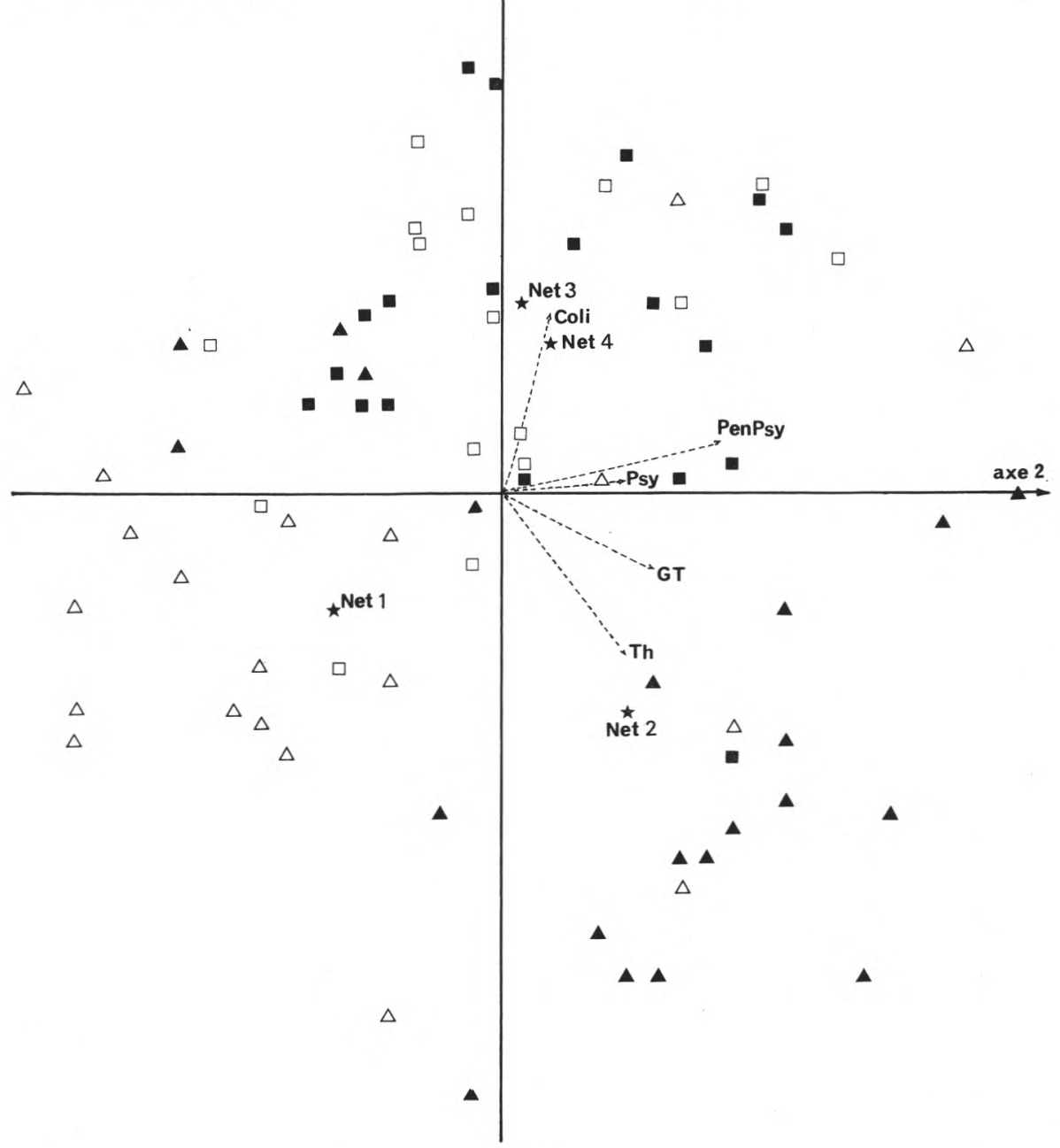

Fig. 1

Classement des échantillons en fonction du niveau et de la nature de la flore microbienne dans le matériel de traite, sur la base d'une analyse discriminante des résultats de numération.

Les flèches en pointillé indiquent le sens des valeurs croissantes pour les flores totales (GT), thermorésistante (Th), psychrotrophe (Psy), psychrotrophe résistante à la pénicilline (PenPsy).

* Résultat moyen dans le système d'axes pour la méthode de nettoyage.

Classification of the samples according to the level and the nature of the bacterial flora on a discriminant analysis of the bacterial counts. 
méthode classique s'est montrée tout aussi performante. Enfin, contrairement à ce qu'ont obtenu Sinclair et Parry (1978), le nettoyage simplifié à l'eau chaude acidifiée conduit à une population microbienne en moyenne plus élevée qu'avec la méthode " E.C.A. " d'origine.

Pour savoir si les diverses méthodes de nettoyage étudiées favorisent des groupes microbiens particuliers, une analyse discriminante portant sur l'ensemble des résultats de dénombrement a été réalisée (Lebart et Fénelon, 1973). Cette analyse revient à rechercher des combinaisons linéaires des variables d'origine (les numérations des divers groupes microbiens) telles qu'à l'intérieur des ensembles constitués par les méthodes de nettoyage, la variabilité intra et inter-ferme soit minimale et que, par contre, les moyennes pour chaque ensemble soient aussi éloignées que possible les unes des autres. La figure 1 montre, pour chaque visite, la position des exploitations dans le plan défini par les axes associés aux deux combinaisons linéaires séparant le mieux les groupes de nettoyage. L'axe 1, assez efficace (pouvoir discriminant de 0,59), oppose les exploitations présentant de fortes teneurs en micro-organismes thermorésistants (et dans une moindre mesure en germes totaux) à celles qui ont un nombre élevé de bactéries coliformes (et dans une plus faible mesure, de micro-organismes psychrotrophes résistants ou non à la pénicilline). Le second axe (pouvoir discriminant de 0,26 ) classe les fermes en fonction du niveau croissant de gauche à droite de la flore pour tous les groupes microbiens et principalement pour la flore totale et la flore psychrotrophe résistante ou non à la pénicilline.

On observe que les méthodes de nettoyage étudiées se distinguent nettement les unes des autres à la fois par le niveau et la nature de la flore microbienne. Si l'on considère le premier axe, avec les méthodes faisant intervenir, au moins une fois par jour, de l'eau chaude acidifiée (E.C.A. et E.C.A. simplifiée), à nombres égaux de germes totaux, on a plus de micro-organismes thermorésistants qu'avec les méthodes basées sur l'utilisation de solutions détergentes à $50-60^{\circ} \mathrm{C}$ (méthode classique avec ou sans alternance de produits). Par contre, avec ces dernières, la flore microbienne est proportionnellement plus riche en bactéries coliformes et en micro-organismes psychrotrophes résistants ou non à la pénicilline. Il ressort de l'examen du second axe que le nettoyage après chaque traite à l'eau chaude acidifiée permet d'obtenir, pour tous les groupes microbiens, des résultats bien meilleurs que sa variante simplifiée. Il n'en est pas de même pour la méthode classique et celle avec alternance de produits acides et basiques dont les points moyens (Net 3 et Net 4) sont proches l'un de l'autre.

Le tableau 2 rassemble les résultats de l'analyse de variance faite pour chaque méthode de nettoyage et chaque groupe microbien. 
$T A B L E A U 2-T A B L E 2$

Analyse de la variance des résultats des dénombrements microbiens effectués sur le second rinçage des installations de traite (calculs effectués sur les logarithmes décimaux)

Variance analysis of results of bacterial counts on second rinses

(computation on $\log _{10}$ base)

\begin{tabular}{|c|c|c|c|c|c|c|}
\hline \multirow[b]{2}{*}{$\begin{array}{l}\text { Méthode } \\
\text { de } \\
\text { nettoyage }\end{array}$} & \multirow[b]{2}{*}{$\begin{array}{l}\text { Source } \\
\text { de } \\
\text { variation }\end{array}$} & \multicolumn{5}{|c|}{ Carrés moyens } \\
\hline & & $\begin{array}{l}\text { Flore } \\
\text { totale }\end{array}$ & $\begin{array}{l}\text { Flore } \\
\text { thermo- } \\
\text { résistante }\end{array}$ & $\begin{array}{c}\text { Flore } \\
\text { psychrotrophe }\end{array}$ & $\begin{array}{l}\text { Flore } \\
\text { psychrotrophe } \\
\text { résistante à la } \\
\text { pénicilline }\end{array}$ & $\begin{array}{l}\text { Bactéries } \\
\text { coliformes }\end{array}$ \\
\hline E.C.A. & $\begin{array}{l}\text { Inter-fermes } \\
\text { Intra-fermes }\end{array}$ & $\begin{array}{l}1,101^{* *} \\
0,089\end{array}$ & $\begin{array}{l}1,120^{* *} \\
0,166\end{array}$ & $\begin{array}{l}1,914^{* *} \\
0,395\end{array}$ & $\begin{array}{l}2,420 * \star \\
0,263\end{array}$ & $\begin{array}{l}0,606 \\
0,462\end{array}$ \\
\hline $\begin{array}{l}\text { E.C.A. } \\
\text { Simplifiée }\end{array}$ & $\begin{array}{l}\text { Inter-fermes (5) } \\
\text { Intra-fermes (15) }\end{array}$ & $\begin{array}{l}2,982^{* *} \\
0,340\end{array}$ & $\begin{array}{l}2,676^{* *} \\
0,173\end{array}$ & $\begin{array}{l}1,140^{*} \\
0,282\end{array}$ & $\begin{array}{l}1,909^{*} \\
0,422\end{array}$ & $\begin{array}{l}0,330 \\
0,200\end{array}$ \\
\hline Classique & $\begin{array}{l}\text { Inter-fermes (4) } \\
\text { Intra-fermes (13) }\end{array}$ & $\begin{array}{l}0,909 \\
0,503\end{array}$ & $\begin{array}{l}0,502 \\
0,369\end{array}$ & $\begin{array}{l}1,100^{*} \\
0,221\end{array}$ & $\begin{array}{l}2,028 * * \\
0,331\end{array}$ & $\begin{array}{l}1,093 \\
0,446\end{array}$ \\
\hline Alternance & $\begin{array}{l}\text { Inter-fermes }(5) \\
\text { Intra-fermes }(13)\end{array}$ & $\begin{array}{l}0,117 \\
0,443\end{array}$ & $\begin{array}{l}0,356 \\
0,759\end{array}$ & $\begin{array}{l}0,036 \\
0,432\end{array}$ & $\begin{array}{l}0,11 \\
0,428\end{array}$ & $\begin{array}{l}0,249 \\
0,721\end{array}$ \\
\hline \multicolumn{2}{|c|}{$\begin{array}{l}\text { Test d'homogénéité des variances } \\
\text { intra-fermes }\end{array}$} & $9,76^{*}$ & $10,86^{*}$ & 1,80 & 0,99 & 5,38 \\
\hline
\end{tabular}

(a) Entre parenthèses : nombre de degrés de liberté.

(b) * ou ** carré moyen inter-ferme significativement plus élevé que le carré moyen intra-ferme aux seuils $\mathrm{P}=0,05$ et $\mathrm{P}=0,01$ respectivement.

(c) Significatif au seuil $\mathbf{P}=0,05$.

(d) Valeur du $\chi^{2}$ de Bartlett (Snedecor et Cochran, 1967). 
TABLEAU 3 - TABLE 3

Comparaison deux à deux des méthodes de nettoyage - Paired comparison of the cleaning methods

\begin{tabular}{|c|c|c|c|c|c|}
\hline \multirow[b]{2}{*}{$\begin{array}{l}\text { Méthodes de nettoyage } \\
\text { comparées }\end{array}$} & \multicolumn{5}{|c|}{ Rapport des moyennes géométriques (a) } \\
\hline & Flore totale & $\begin{array}{l}\text { Flore } \\
\text { thermorésistante }\end{array}$ & $\begin{array}{c}\text { Flore } \\
\text { psychrotrophe }\end{array}$ & $\begin{array}{l}\text { Flore } \\
\text { psychrotrophe } \\
\text { pénícillino- } \\
\text { résistante }\end{array}$ & $\begin{array}{l}\text { Bactéries } \\
\text { coliformes }\end{array}$ \\
\hline E.C.A. vs E.C.A. simplifiée & $\begin{array}{r}5,24 \\
(1,62)\end{array}$ & $\begin{array}{c}5,53 \\
(1,73)\end{array}$ & $\begin{array}{c}2,99 \\
(1,23)\end{array}$ & $\begin{array}{c}7,82 \\
(1,94)\end{array}$ & $\begin{array}{c}1,09 \\
(0,17)\end{array}$ \\
\hline E.C.A. vs classique & $\begin{array}{r}1,18 \\
(0,23)\end{array}$ & $\begin{array}{c}3,02 \\
(1,66)\end{array}$ & $\begin{array}{c}1,87 \\
(0,69)\end{array}$ & $\begin{array}{c}5,57 \\
(1,54)\end{array}$ & $\begin{array}{c}3,24 \\
(1,69)\end{array}$ \\
\hline Classique avec $v s$ sans alternance & $\begin{array}{c}3,54 \\
(2,31)\end{array}$ & $\begin{array}{l}3,70 \\
(2,63)^{*}\end{array}$ & $\begin{array}{c}2,11 \\
(1,29)\end{array}$ & $\begin{array}{l}1,91 \\
(0,82)\end{array}$ & $\begin{array}{c}3,15 \\
(1,83)\end{array}$ \\
\hline $\begin{array}{l}\text { E.C.A. simplifiée vs classique } \\
\text { avec alternance }\end{array}$ & $\begin{array}{c}1,75 \\
(0,63)\end{array}$ & $\begin{array}{c}4,51 \\
(1,71)\end{array}$ & $\begin{array}{c}1,32 \\
(0,51)\end{array}$ & $\begin{array}{c}1,36 \\
(0,43)\end{array}$ & $\begin{array}{l}11,07 \\
(6,15)^{* *}\end{array}$ \\
\hline \multicolumn{6}{|c|}{$\begin{array}{l}\text { (a) Voir tableau } 1 \text { pour les moyennes. } \\
\text { (b) Entre parentèses : valeur de } t \text { du test de Student (Snedecor et Cochran, 1967). } \\
\text { * ou ** significatif aux seuils } P=0,05 \text { et } P=0,01 \text { respectivement. }\end{array}$} \\
\hline
\end{tabular}


Dans le cas du lavage à l'eau chaude acidifiée (E.C.A. et E.C.A. simplifiée), la variabilité des résultats d'une exploitation à l'autre est, sauf pour les bactéries coliformes, très nettement supérieure aux variations entre les résultats d'une même ferme. Cela est probablement imputable à des différences de « caractéristiques géométriques » (conception ou montage plus ou moins soignés) entre les installations de traite concernées (Richard, 1981 a). Par contre, avec les deux autres techniques de nettoyage (exception faite, dans le cas de la méthode classique, des micro-organismes psychrotrophes et psychrotrophes pénicillinorésistants) les différences entre exploitations n'apparaissent pas plus importantes qu'elles ne le sont d'une visite à l'autre dans une même ferme; lorsqu'il y a alternance de produits, elles sont même beaucoup plus faibles, quel que soit le groupe microbien. Cela ne veut pas dire que les caractéristiques géométriques du matériel de traite soient sans incidence sur les résultats de ces méthodes de nettoyage, mais que cette incidence peut être masquée par une plus grande variabilité des résultats obtenus dans une même exploitation. En effet, pour les germes totaux et les micro-organismes thermorésistants, nous avons mis en évidence une hétérogénéité significative des variances " intrafermes ", celles correspondant au nettoyage classique ou avec alternance étant les plus élevées. On notera enfin, dans les exploitations où se pratique l'alternance journalière de produits détergents alcalins et acides à $50-60^{\circ} \mathrm{C}$, une plus grande irrégularité du " pouvoir contaminant " des installations de traite au cours du temps que dans les autres fermes (variance intra-ferme toujours supérieure à 0,4 ) (test de Bartlett, Snedecor et Cochran, 1967). Finalement pour comparer entre elles les quatre méthodes de nettoyage, nous avons retenu comme estimateur de la variabilité des résultats moyens de chacune d'elles, le quotient du carré moyen « intra-ferme » (tab. 2) par le nombre d'observations concourant à la moyenne (Snedecor et Cochran, 1967). On constate (tab, 3) qu'il n'existe généralement pas de différence significative entre les méthodes prises deux à deux. En particulier, si le nettoyage simplifié à l'eau chaude acidifiée conduit à une population microbienne dans les installations de traite en moyenne plus élevée qu'avec la méthode "E.C.A. " d'origine (plus de 5 fois pour la flore totale, les germes psychrotrophes pénicillinorésistants et les micro-organismes thermorésistants), cette différence n'est cependant pas significative. C'est sans doute la conséquence d'une variabilité importante des résultats d'une exploitation à l'autre liée, vraisemblablement, aux caractéristiques géométriques du matériel de traite.

\section{CONCLUSIONS}

Compte tenu des exigences actuelles en matière de qualité bactériologique du lait, les performances du nettoyage biquotidien à 
l'eau chaude acidifiée sont le plus souvent très satisfaisantes (apport maximum inférieur à 10000 micro-organismes totaux par ml de lait ou inférieur à 1000 , pour la flore thermorésistante). Toutefois ces performances, dans certaines fermes, sont moins bonnes, du fait probablement de la conception ou du montage défectueux des machines à traire.

Le remplacement de l'eau chaude acidifiée par un simple rinçage après la traite du soir (nettoyage E.C.A. simplifié) présente un intérêt économique indéniable et peut être envisagé avec certains types d'installations qui restent à définir. Ainsi dans l'une des exploitations, la flore totale du matériel de traite était en moyenne de 1400 germes $/ \mathrm{ml}$, avec des valeurs extrêmes de 100 à 7000 germes $/ \mathrm{ml}$, ce qui est tout à fait acceptable.

Le nettoyage classique permet en général d'obtenir des résultats acceptables. Ceux-ci, malgré tout, présentent des variations « intrafermes " significativement plus importantes que dans le cas des deux méthodes précédentes.

L'alternance journalière de produits détergents alcalins et acides, discutable sur le plan économique, l'est aussi sur celui des performances. En effet, sans que l'on puisse l'expliquer, elle est à l'origine d'une grande irrégularité dans le temps du " pouvoir contaminant " des machines à traire.

La nature de la flore microbienne des installations de traite est plus influencée par la méthode de nettoyage que par la conception ou l'état du matériel qui ne font, le cas échéant, qu'amplifier les tendances propres à chaque méthode. C'est ce que l'un de nous avait déjà observé (Richard, 1981 a). Le recours à l'eau chaude acidifiée entraîne une flore microbienne proportionnellement plus riche en micro-organismes thermorésistants; au contraire, l'utilisation de détergents alcalins à $50-60^{\circ} \mathrm{C}$ favorise les micro-organismes psychrotrophes pénicillinorésistants. Cependant, on a montré que ces différences n'affectaient pas significativement la « qualité de conservation » du lait cru à basse température (Richard 1981 b), cela sans doute parce que les Pseudomonas psychrotrophes qui se multiplient activement dans ce milieu à une température comprise entre 4 et $5^{\circ} \mathrm{C}$ ont une autre origine. D'un point de vue plus théorique, la présence dans les installations de traite nettoyées deux fois par jour à l'eau chaude acidifiée, de bactéries à Gram négatif (coliformes ou Pseudomonas), peut surprendre, en raison de la faible résistance de ces micro-organismes à la chaleur. Ces bactéries peuvent provenir de certaines parties de l'installation étrangères au circuit du lait, et donc non ébouillantées, mais communiquant avec lui (connection avec le circuit de vide par exemple). Elles peuvent aussi résulter d'un défaut d'étanchéité des installations sous vide permettant la pénétration, dans le circuit du lait, d'eau sale qui lui est extérieure. 
Le travail que nous avons réalisé montre la nécessité de déterminer l'efficacité, à long terme, des méthodes de nettoyage au cours d'essais dans des exploitations ne bénéficiant pas d'une surveillance particulière. A l'occasion de ceux-ci, il est tout aussi important, sur le plan méthodologique, de s'attacher à la variabilité « entre fermes 》 et " intra-fermes " des résultats obtenus qu'aux performances moyennes de chacune des méthodes. Ceci implique de conduire ces essais dans un nombre suffisant d'exploitations (au moins 6 par type de nettoyage étudié) et sur une période de durée convenable ( 6 mois au minimum). Une technique de nettoyage sera alors considérée comme intéressante à vulgariser si elle se justifie économiquement et qu'elle est fiable (résultats régulièrement bons dans l'ensemble des fermes de l'essai).

\section{Remerciements}

Cette étude a été réalisée avec le concours de l'Union Laitière de la Meuse et du Contrôle Laitier de la Sarthe. Nous tenons à remercier les organismes ainsi que les producteurs de lait de ces deux départements qui se sont prêtés de bonne grâce à nos contrôles.

\section{Bibliographie}

Chatelin (Y. M.), Richard (J.) (1981), - Etude de quelques cas de contaminations microbiennes importantes du lait à la ferme. Lait, 61, 80-94.

Clough (P.), AKam (D.), CANT (D.) (1965). - Circulation cleaning of pipeline milking machines with boiling water. Nird Paper $\mathrm{n}^{\circ} 2939$.

Gilchrist (J. E.), Campbell (J. E.), Donnelly (C. B.), Peeler (J. T.), Delaney (J. M.) (1973). - Spiral plate method for bacterial determination. Appl. Microbiol., $25,244-252$.

LEBART (L.), FÉNÉLON (J. P.) (1973). - Statistique et informatique appliquées. Dunod. $2^{*}$ éd., Paris.

Palmer (J. M. A.), Fleming (M. G.), Mulcamy (M. J.), Power (L.), Moran (M. A.), CoNNOLY (S.) (1970). - Enlarged field trial of boiling water-citric acid cleaning of bucket milking machines. Irish J. Agr. Res., 9, 179-186.

Piton (C.), RichaRd (J.) (1982). - Causes de contamination microbienne d'importance moyenne du lait dans un groupe de fermes de la région de Rennes. Lait, 62, 67-74.

Richard (J.) (1974). - Nettoyage simplifié du matériel de traite en pot. Revue Laitière Française, 320, 325-338.

RichaRd (J.) (1980). - Influence de l'agitation du lait cru sur les résultats de dénombrement de sa flore totale à l'aide d'une anse calibrée. Lait, 60, 211-225.

RICHARD (J.) (1981 a). - Bacteriological aspects of cold cleaning with an iodophor of milk pipeline installation. J. appl. Bact., 50, 229-238.

RICHARD (J.) (1981 b). - Influence de diverses méthodes de nettoyage des machines à traire sur la "qualité de conservation " du lait cru à basse température. Lait, 61, 354-369.

RICHARD (J.) (1981 c). - Bacteriological examination of pipeline milking machine by rinsing the entire system. J. appl. Bact., 50, 433-442. 
Sinclair (G.), PARRY (D. R.) (1978). - Nettoyage une fois par jour des installations de traite à transfert à l'eau bouillante acidifiée. $X X^{\mathrm{e}}$ Congrès International de Laiterie, 130-131.

SNedecor (G. W.), Cochran (W. G.) (1967). - Statistical methods 6 th ed. The Jowa State University Press, Ames, Jowa, U.S.A.

StraKa (R. P.), StoKes (J. L.) (1957). - Rapid destruction of bacteria in commonly used diluents and its elimination. Appl. Microbiol., 5, 21-25. 